\title{
Base Pair
}

National Cancer Institute ( $\mathrm{NCl})$

\section{Source}

National Cancer Institute (NCI). Base pair.

Two nitrogen-containing bases pair together between double-stranded DNA; only specific combinations of these bases (e.g., adenine with thymine; guanine with cytosine) are possible, a fact which facilitates accurate DNA replication; when quantified (e.g., 8 base pairs, or bp), this term refers to the actual number of base pairs in a sequence of nucleotides. 